Revue internationale P.M.E.

Économie et gestion de la petite et moyenne entreprise

Revue

internationale

PME

\title{
Implantation d'un programme de formation en entrepreneuriat et ressources interorganisationnelles régionales : le cas du Québec
}

\section{Jean-Pierre Béchard}

Volume 11, numéro 4, 1998

URI : https://id.erudit.org/iderudit/1009054ar

DOI : https://doi.org/10.7202/1009054ar

Aller au sommaire du numéro

Éditeur(s)

Presses de l’Université du Québec

ISSN

0776-5436 (imprimé)

1918-9699 (numérique)

Découvrir la revue

Citer cette note

Béchard, J.-P. (1998). Implantation d'un programme de formation en entrepreneuriat et ressources interorganisationnelles régionales : le cas du Québec. Revue internationale P.M.E., 11(4), 105-123.

https://doi.org/10.7202/1009054ar
Résumé de l'article

Cet article s'intéresse à la nature et aux rôles des ressources communautaires qui appuient les programmes de formation en entrepreneuriat offerts en contexte scolaire. À partir d'une analyse en profondeur du processus d'implantation d'un programme de formation en entrepreneuriat dans 16 commissions scolaires du Québec, l'auteur met au jour, d'une part, les 10 étapes du processus d'implantation du programme et, d'autre part, les 13 catégories d'organisations qui soutiennent la formation étudiée. Ces résultats permettent la construction d'une mesure de la valeur des interactions socio-organisationnelles (VISO) pour mieux saisir le degré d'ouverture de l'école vers son milieu quand il est question de formation en entrepreneuriat L'article se termine par un débat sur l'utilité d'un tel indicateur. 


\title{
Implantation d'un programme de formation en entrepreneuriat et ressources interorganisationnelles régionales: le cas du Québec
}

\author{
Jean-Pierre BÉCHARD \\ École des Hautes Études commerciales, Montréal
}

\section{MOTS CLÉS}

\section{Programme de formation - Entrepreneuriat - PME - Éducation Ressources régionales - Interactions - Commission scolaire}

\begin{abstract}
RÉSUMÉ
Cet article s'intéresse à la nature et aux rôles des ressources communautaires qui appuient les programmes de formation en entrepreneuriat offerts en contexte scolaire. À partir d'une analyse en profondeur du processus d'implantation d'un programme de formation en entrepreneuriat dans 16 commissions scolaires du Québec, l'auteur met au jour, d'une part, les 10 étapes du processus d'implantation du programme et, d'autre part, les 13 catégories d'organisations qui soutiennent la formation étudiée. Ces résultats permettent la construction d'une mesure de la valeur des interactions socio-organisationnelles (VISO) pour mieux saisir le degré d'ouverture de l'école vers son milieu quand il est question de formation en entrepreneuriat. L'article se termine par un débat sur l'utilité d'un tel indicateur.
\end{abstract}

\section{L'AUTEUR}

Jean-Pierre Béchard est détenteur d'un doctorat en management et professeur en entrepreneuriat et PME à l'École des Hautes Études commerciales de Montréal. Il est aussi chercheur associé à la Chaire Maclean Hunter en entrepreneuriat de cette même institution. Ses travaux portent notamment sur l'analyse des programmes de formation en entrepreneuriat et PME en contexte scolaire et communautaire à travers le monde. Adresse: 3000 , chemin de la Côte Sainte-Catherine, Montréal, Canada, H3T 2A7, Téléphone : (514) 340-6379, Télécopieur : (514) 340-5635, Courriel : jean-pierre.bechard@hec.ca 


\begin{abstract}
This article deals with the nature and roles of community resources which support training programs in entrepreneurship in the school system. The author made an in-depth analysis of the implementation process of an entrepreneurship training program in 16 school boards in Quebec and thus was able to show the 10 steps in the implementation process and the 13 categories of organizations offering support to such a training program. These results lead to the making of an indicator of the value of the interactions between society and the organization (VISO) which in turn makes it possible to understand the degree of openness of a school towards its environment on the question of entrepreneurship training. The article ends with a debate on the usefulness of such an indicator.
\end{abstract}

\title{
RESUMEN
}

Este artículo trata sobre la naturaleza y los roles de los recursos de la comunidad que apoyan los programas de formación en espíritu empresarial ofrecidos en contexto escolar. A partir de un análisis profundo del proceso de implantación de un programa de formación en espíritu empresarial en 16 comisiones escolares de Québec, el autor presenta inicialmente las diez etapas del proceso y luego las 13 categorías de organizaciones que soportan la formación. Los resultados permiten la construcción de un indicador del valor de las interacciones socio-organizacionales (VISO) que permite comprender mejor el grado de apertura de la escuela hacia su medio, cuando se trata de formación en espíritu empresarial. El artículo concluye con un debate sobre la utilidad de este tipo de indicador.

\section{Introduction}

L'enseignement en entrepreneuriat soulève un débat important dans les écoles, les collèges et les universités. Peut-on laisser à l'école seule la responsabilité de sensibiliser les personnes à la carrière en entrepreneuriat, ainsi que l'enseignement des étapes de la création et du développement d'une petite entreprise ? Doit-on utiliser toutes les ressources éducatives du milieu pour s'assurer de la qualité des programmes de formation en entrepreneuriat ? Ces différentes interrogations concernant la place des organisations scolaires et communautaires dans l'enseignement en entrepreneuriat sont à la base même de la problématique de cette recherche. Or, comme cet enjeu concerne bon nombre de formateurs, il nous semble opportun d'y réfléchir et d'y apporter un éclairage nouveau.

Tout au long de cet article, nous ferons référence au concept de programme et plus spécifiquement de programme de développement en entrepreneuriat (PDE). Un tel programme est un ensemble d'enseignements formalisés qui informent, forment et éduquent toute personne intéressée à participer au développement socioéconomique par l'intermédiaire d'un projet de sensibilisation, de création et de développement d'entreprises et de formateurs. Fort de cette définition commune, jetons maintenant un coup d'œil sur ce que la littérature en entrepreneuriat / PME et éducation rapporte sur le sujet des ressources éducatives qui gravitent autour de ces PDE. 


\section{Revue de la documentation}

Plusieurs travaux de recherches s'intéressent à la nature et aux rôles des ressources scolaires et communautaires impliquées dans l'enseignement en entrepreneuriat. Ce bassin de ressources éducatives est donc un élément jouant un rôle crucial dans le développement socioéconomique des milieux.

\section{Ressources scolaires}

Une des pierres d'assises du système scolaire en entrepreneuriat est sans contredit le formateur. Au primaire et au secondaire, les enseignants d'éducation économique sont majoritairement formés sur le tas et ont pour la plupart mis au point des programmes en entrepreneuriat avec l'appui de fondations et quelquefois des ministères d'éducation nationale (Robinson et Christensen, 1992). Au niveau collégial et universitaire, nous commençons à identifier de plus en plus de spécialistes en entrepreneuriat. Katz $(1991,1993)$ a tenté de cerner ce phénomène en dégageant le nombre de postes universitaires de prestige, tels les chaires et les professorship, dans le champ de l'entrepreneuriat, de la libre entreprise et dans les domaines connexes telle l'économie. En date de 1993, il dénombre plus de 123 postes universitaires de prestige dont 32 sont toujours vacants. Cet état de fait laisse entendre, d'une part, que la carrière de professeur universitaire en entrepreneuriat devient plus valorisante et permet de gravir plus facilement les échelons institutionnels et que, d'autre part, il y a encore très peu d'étudiants qui font leur doctorat en entrepreneuriat. Mais, dans son article sur l'infrastructure universitaire européenne, Carsrud (1991) constate l'inverse de la situation américaine : peu de possibilités de faire carrière en entrepreneuriat, mais plus d'étudiants inscrits au programme européen de doctorat en entrepreneuriat et petite entreprise.

Les ressources scolaires au niveau collégial et universitaire comprennent aussi les centres de recherches, les fondations privées et les revues spécialisées. Les centres de recherches sont considérés comme un apport important dans l'infrastructure entourant les programmes de développement en entrepreneuriat (Kierulff, 1985 ; Robinson et Haynes, 1991 ; Sandberg et Gatewood, 1991). Dans sa dernière étude, Katz (1993) dénombre 54 centres américains, canadiens et britanniques incluant ceux qui se consacrent à l'entreprise familiale. En outre, Gatewood, Miranda et Hoy (1990) analysent les actions de 12 fondations privées qui s'intéressent particulièrement à l'entrepreneuriat. En plus de cette liste, Katz (1993) intègre les fondations Kellogg, Ford et Kauffman qui soutiennent la recherche et l'enseignement en entrepreneuriat. Les publications à caractère pratique et scientifique font aussi partie de ces ressources éducatives. Katz (1993) recense 25 revues en entrepreneuriat-PME et dans les domaines connexes. 
Nous pouvons résumer l'état de la question des ressources scolaires de la façon suivante : du côté du primaire et du secondaire, malgré la croissance des initiatives locales par de nombreux professeurs venant d'autres disciplines, il y a très peu d'engagement au niveau des ressources scolaires à l'exception de certains groupes de promotion voués au développement de l'entrepreneuriat. Il ne faut certes pas s'étonner de cette réalité. Malgré l'avis du Conseil supérieur de l'éducation (1989), le développement socioéconomique régional reste un choix à préciser pour les commissions scolaires. Quant au milieu universitaire, il est en pleine expansion avec une croissance vertigineuse des postes universitaires de prestige, des centres de recherches et des publications, le tout abondamment subventionné par le gouvernement et surtout par l'apport financier majeur de fondations privées.

\section{Ressources communautaires}

L'information sur les ressources communautaires qui gravitent autour des programmes de formation en entrepreneuriat fait état de nombreuses infrastructures qui ne sont pas de nature scolaire mais qu'il importe de mentionner à ce stade-ci. Pensons tout d'abord aux agences internationales de développement dont le Bureau international du travail (Brockhaus Sr., 1991 ; Gibb et Manu, 1990), aux organisations professionnelles (Christy et Jones, 1982; Katz, 1991), aux incubateurs (Carsrud, 1991), aux gouvernements fédéraux et locaux (Dana, 1990; Solomon et Carney, 1985) et aux Chambres de commerce (Leclerc, 1985). De plus, il y a les organisations étudiantes (Welsch, 1993), les entrepreneurs (Delanay, 1988), les organisations communautaires régionales (Carsrud, 1991 ; Welsch, 1993). N'oublions pas aussi les institutions financières et religieuses, les médias (Welsch, 1993), les agences de publicité (Dart et Pendleton, 1984) pour terminer avec les corporations de développement économique et les groupes de promotion de l'entrepreneuriat (Robinson et Christensen, 1992).

Ce débat concernant les ressources éducatives qui soutiennent les programmes de formation en entrepreneuriat met en lumière, d'une part, la nature et le rôle des organisations scolaires et communautaires dédiées à l'entrepreneuriat et, d'autre part, leur diversité respective. Mais, s'il y a ampleur et diversité des ressources éducatives, cela ne veut pas dire pour autant qu'il y a qualité et efficacité des programmes de formation en entrepreneuriat. Ce présent article cherche à pousser plus loin cette réflexion en voulant répondre à la question suivante : comment qualifier les interactions entre les ressources éducatives et, plus précisément, entre l'organisation formatrice (école, collège, université ou autre) et les ressources du milieu ? En effet, il ne suffit pas d'être entouré de ressources éducatives, encore faut-il les utiliser aux endroits stratégiques et avec une intensité telle que la formation puisse en bénéficier. Nous pensons que l'étude des interactions entre l'organisation formatrice et les autres organisations du milieu est importante pour la compréhension des 
programmes de développement en entrepreneuriat et qu'elle constitue une voie de recherche intéressante.

\section{Cadre théorique}

Comment peut-on qualifier les liens tissés entre l'école et le milieu ? Il est important de mentionner que l'on ne peut mettre sur le même pied toutes les organisations qui interagissent avec l'école. Certaines sont peu sympathiques à la cause de la formation tandis que d'autres s'y impliquent à fond.

Ainsi, toute forme d'interaction entre une organisation et une autre peut varier en fonction de la fréquence $(\mathrm{F})$ des contacts interorganisationnels et de l'intensité de ces mêmes liens (I). On peut raisonnablement penser que la multiplication de $\mathrm{F} \times \mathrm{I}$ offrirait une indication juste de la valeur de l'interaction entre une organisation et une autre. Sur le plan théorique, cette mesure $(\mathrm{F} \times \mathrm{I})$ prend appui sur les travaux d'Aldrich (1976) et de Hall (1987) quant au nombre de contacts entre les organisations et sur ceux de Marrett (1971) et d'Aldrich (1976) sur l'intensité des ressources investies par les organisations lors de l'interaction.

Nous pouvons opérationaliser les interactions qui nous intéressent en identifiant, d'une part, les ressources communautaires qui gravitent autour de l'école et, d'autre part, l'intensité de ces interactions. Les recherches mentionnées ci-haut dégagent trois niveaux que l'on peut accorder à une interaction école-milieu. Le niveau 1 désigne un contact de l'école envers le milieu : c'est la dimension de l'information. Le niveau 2 est attribué à une interaction plus intense, où non seulement l'école prend contact, mais où l'organisation concernée donne son appui à un projet, est solidaire de la formation. Enfin, le niveau 3 renvoie à une interaction où les deux partenaires, école et organisation, produisent un travail qui vise la formation, et ce, à part l'étape du processus d'implantation. Nous identifions donc trois niveaux d'intensité d'une interaction.

Figure 1

Niveau des interactions école-milieu

\begin{tabular}{|c|c|c|}
\hline École & $\begin{array}{l}\text { Niveau 1 } \\
\qquad \rightarrow \rightarrow \rightarrow \rightarrow \rightarrow \rightarrow \rightarrow \rightarrow \rightarrow \\
\text { Information }\end{array}$ & Milieu \\
\hline École & $\begin{array}{l}\text { Niveau } 2 \\
\leftarrow \leftarrow \leftarrow \leftarrow \leftarrow \leftarrow \leftarrow \leftarrow\end{array}$ & Milieu \\
\hline École & 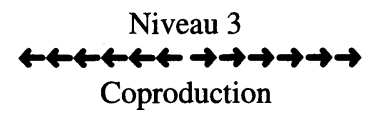 & Milieu \\
\hline
\end{tabular}


En résumé, nous pouvons mesurer la valeur des interactions socioorganisationnelles (VISO) en multipliant la fréquence des interactions école-milieu par l'intensité de ces interactions. On peut raisonnablement penser qu'un VISO faible indique qu' une organisation formatrice est relativement fermée sur son milieu tandis qu'un VISO élevé indique une grande ouverture de l'école sur son milieu.

\section{Méthodologie}

À la suite d'un protocole d'entente entre le ministère de l'Éducation du Québec (MEQ) et la Chaire Maclean Hunter en entrepreneuriat de l'École des Hautes Études commerciales de Montréal, nous avons procédé durant la période 1992-1993 à l'analyse en profondeur du processus d'implantation du nouveau programme de la Direction générale de la formation professionnelle (DGFP) du MEQ, programme intitulé «Lancement d'une entreprise ». Ce programme donne droit à une attestation de spécialisation professionnelle (ASP) reconnue par le MEQ.

La durée du programme est de 330 heures; de ce nombre 220 heures sont consacrées à l'acquisition de compétences liées directement à la maîtrise des tâches d'un entrepreneur ou d'une entrepreneure et de 110 heures à l'acquisition de compétences plus larges. Le programme est divisé en six modules dont la durée varie de 30 heures à 120 heures. Cette durée comprend le temps requis pour l'évaluation des apprentissages aux fins de la sanction des études et pour l'enseignement correctif (DGFP, 1991c). Voici la répartition des heures selon les six modules de ce programme:

1. Entrepreneuriat et formation

2. Définition d'un projet d'entreprise

3. Élaboration d'un plan d'affaires

4. Application des notions de gestion d'entreprise

5. Applications des techniques de vente

6. Négociation du financement d'une entreprise
30 heures

30 heures

120 heures

75 heures

30 heures

45 heures

La conception de ce programme a été l'œuvre commune d'experts en éducation du MEQ, d'entrepreneurs en exercice et de formateurs reconnus en entrepreneuriat. Ce comité a validé le contenu des six modules ainsi que le nombre d'heures à allouer pour chacun d'entre eux (DGFP, 1991b). Puis, la DGFP a présenté le 8 octobre 1991 les grandes lignes de ce programme pilote à plus de 47 commissions scolaires du Québec qui se montraient intéressées à obtenir cette carte d'enseignement. De ce nombre, plus d'une trentaine ont présenté officiellement le document de demande. Un choix a été fait par le MEQ sur la base de critères inscrits dans les orientations de ce programme et 16 commissions scolaires furent retenues en avril 1992. Le tableau 1 présente les noms des commissions scolaires choisies ainsi que leur région respective. 
TABLEAU 1

Liste des commissions scolaires

offrant le programme «Lancement d'une entreprise » 1992-1993

\begin{tabular}{|c|c|c|c|}
\hline $\begin{array}{l}\text { Commission } \\
\text { scolaire }\end{array}$ & Région & $\begin{array}{l}\text { Commission } \\
\text { scolaire }\end{array}$ & Région \\
\hline $\begin{array}{l}\text { Vallée-de-la-Matapédia } \\
\text { (MATA) }\end{array}$ & Bas-St-Laurent & Mauricie (MAUR) & Mauricie-Bois Francs \\
\hline Beauport (BEAU) & Québec & Ste-Croix (STCR) & Montréal \\
\hline Draveurs (DRAV) & Outaouais & Harricana (HARR) & $\begin{array}{l}\text { Abitibi- } \\
\text { Témiscamingue }\end{array}$ \\
\hline Bersimis (BERS) & Côte-Nord & $\begin{array}{l}\text { Chutes-de-la- } \\
\text { Chaudière (CHUT) }\end{array}$ & $\begin{array}{l}\text { Chaudière- } \\
\text { Appalaches }\end{array}$ \\
\hline $\begin{array}{l}\text { Chomedey-Laval } \\
\text { (CHOM) }\end{array}$ & Laval & Le Gardeur (LEGA) & Lanaudière \\
\hline Laurentides (LAUR) & Laurentides & Châteauguay (CHA) & Montérégie \\
\hline Découvreurs (DECO) & Québec & CECM (CECM) & Montréal \\
\hline Chicoutimi (CHIC) & $\begin{array}{l}\text { Saguenay- } \\
\text { Lac-St-Jean }\end{array}$ & Portneuf (PORT) & Québec \\
\hline
\end{tabular}

Nous avons procédé à deux collectes de données auprès des 16 enseignantscoordonnateurs du programme répartis dans autant de commissions scolaires du Québec. La première a eu lieu au cours du mois de novembre 1992 et la seconde en avril 1993.

Comme notre première collecte se voulait de nature inductive, nous avons utilisé la méthode de l'entrevue semi-structurée. La question principale s'énonçait comme suit : «Parlez-moi de l'implantation du programme dans votre commission scolaire? » Les questions sous-jacentes étaient les suivantes : «Quelles sont les étapes d'implantation du programme depuis le début jusqu'à aujourd'hui ? Quel est le rôle de chaque acteur scolaire et communautaire en rapport avec les étapes d'implantation?»

Entre la première et la deuxième tournée, nous avons analysé le contenu des 16 dossiers (entrevues et documentation écrite). Les résultats nous ont incité à élaborer un questionnaire sur les réseaux scolaires et communautaires eu égard au programme de formation. Ce questionnaire présente les 10 étapes du processus d'implantation du programme tel que décodé lors des entrevues de la première tournée. De plus, pour chaque étape préalablement définie, on retrouve l'ensemble des acteurs scolaires et communautaires qui s'intéressent au programme. 
Lors de la deuxième tournée, en hiver 1993, nous avons présenté ce questionnaire à nos interlocuteurs (enseignants-coordonnateurs) de l'automne 1992. Rappelons que cette analyse inductive-déductive permet de vérifier la fiabilité des entrevues semistructurées et la validité des données recueillies. De plus, le fait que nous ayons promis la confidentialité totale et donné l'information quant à l'utilisation des données et l'assurance de l'accessibilité aux résultats finaux, avant tout pour satisfaire des considérations éthiques, contribue à garantir la validité des données.

Dans le cadre de cet article, nous portons notre attention sur les ressources communautaires, laissant délibérément de côté les données sur les ressources scolaires. L'analyse des données provenant de ce questionnaire a permis de construire un indice, le VISO ou valeur des interactions socio-organisationnelles. Rappelons qu'un VISO faible indique que le programme de formation est fermé sur lui-même tandis qu'un VISO élevé reflète une ouverture très grande du programme sur le milieu. Ce continuum représente le spectre des conceptions de l'éducation. Mentionnons enfin que la fiabilité de cette mesure a été éprouvée lors d'une rencontre à la fin de l'année scolaire avec les 16 enseignants-coordonnateurs. Nous leur avons demandé de se positionner eux-mêmes dans un des trois groupes de VISO (faible, moyen, élevé) : nos résultats ont été identiques dans 15 cas sur 16.

\section{Résultats}

Nous présentons ici les résultats reliés au processus d'implantation du programme de formation, ainsi que ceux reliés aux acteurs du milieu qui ont contribué à cette formation en entrepreneuriat.

\section{Le processus d'implantation}

D'après l'étude approfondie des résultats des entrevues semi-structurées passées auprès des 16 enseignants-coordonnateurs, le processus d'implantation de ce programme se divise en 10 étapes bien distinctes. Voyons tout d'abord une brève définition de chacune d'entre elles :

\section{Étape 1: obtention de l'ASP}

Après avoir posé le diagnostic de ses forces et de ses faiblesses et fait le constat des menaces et des appuis qui l'entourent, la commission scolaire décide de présenter un projet à la Direction générale de la formation professionnelle et obtient finalement le droit d'offrir le programme «Lancement d'une entreprise » pour l'année 1992-1993.

\section{Étape 2 : planification budgétaire}

Processus écrit de prévision des coûts financiers que devra assumer la commission scolaire et la DGFP pour gérer ce programme. Ces coûts incluent l'achat de mobilier, d'appareillage, d'outillage, de matières premières et de services de soutien, ainsi 
que de matériel didactique. À cela, il faut ajouter les salaires des gens impliqués dans ce programme.

\section{Étape 3: organisation des locaux}

Étape visant la mise en service d'un local aménagé selon les besoins des candidats et les contraintes de la commission scolaire. Cela concerne autant la disposition du matériel et du local que les conditions d'utilisation.

\section{Étape 4 : choix du coordonnateur}

Processus de recrutement, de sélection, d'embauche et de perfectionnement de l'enseignant-coordonnateur.

\section{Étape 5 : promotion du programme}

Étape de sensibilisation des gens de la commission scolaire et de la communauté au nouveau programme «Lancement d'une entreprise».

\section{Étape 6: recrutement des candidats}

Étape qui consiste à utiliser un ensemble d'outils promotionnels (moyens écrits ou visuels) pour attirer le maximum de candidats potentiels lors des soirées d'information.

\section{Étape 7 : sélection des candidats}

Processus de jugement par critères qui vise à retracer parmi les candidatures potentielles les projets les plus susceptibles de bénéficier du programme.

\section{Étape 8 : planification pédagogique}

Démarche qui consiste à structurer les objectifs, les éléments de contenu, les stratégies d'enseignement, les activités d'apprentissage et les critères de passation en séquences cohérentes.

\section{Étape 9 : enseignement en classe}

Suite de rencontres entre les formateurs et les candidats pour tenter d'apprendre et de comprendre les connaissances, les habiletés et les attitudes nécessaires à la création d'une entreprise.

\section{Étape 10: évaluation des apprentissages}

Processus de sanction des apprentissages selon des critères préétablis, individuellement ou conjointement, par le formateur, le candidat ou le ministère de l'Éducation.

Fait important à noter, si les commissions scolaires ont passé ces 10 étapes, l'ordre dans lequel elles ont été vécues diffère d'une région à l'autre. La définition des 10 étapes du processus d'implantation prépare maintenant le terrain à l'identification des acteurs clés et de leurs rôles respectifs. Ces données proviennent des questionnaires passés lors de la deuxième tournée d'entrevues. 


\section{Les acteurs clés}

Dans l'ensemble, les 16 commissions scolaires participantes ont pris contact avec 96 organisations différentes. Ces organisations se regroupent en 13 catégories distinctes comme nous le voyons au tableau 2.

TABLEAU 2

Les commissions scolaires et leurs contacts socio-organisationnels

\begin{tabular}{lll}
\hline $\begin{array}{l}\text { Institutions financières } \\
\text { (IF) }\end{array}$ & $\begin{array}{l}\text { Corporations de développement } \\
\text { (CD) }\end{array}$ & $\begin{array}{l}\text { Groupes de soutien } \\
\text { (GS) }\end{array}$ \\
\hline Banque de Montréal & CODE & SAJE \\
Banque Nationale & DECEC & CAE \\
Caisse Populaire & Commissariat industriel & Incubateur \\
Banque Fédérale de développement & SEEP & SDEL \\
Fédération des Caisses & COPEM & Jeunes Entreprises \\
Banque Royale & CDEC & Fondation de l'Entrepreneurship \\
Banque Canadienne Impériale & CDL & Conseil Économique \\
de Commerce & Agence socioéconomique & \\
Banque Nova Scotia & CDEB & \\
& CODICEM & \\
\hline
\end{tabular}

Organisations scolaires

Organisations communautaires

(OS) (OC)

Cégep Carrefour Emploi-Jeunesse

Autres commissions scolaires $\quad$ Groupe communautaire

Organisme ethnique

CLIP

YMCA

Organisme culturel

\begin{tabular}{lll}
\hline $\begin{array}{l}\text { Gouvernement fédéral } \\
\text { (GF) }\end{array}$ & $\begin{array}{l}\text { Fonds de financement } \\
\text { (FF) }\end{array}$ & $\begin{array}{l}\text { Gouvernement municipal } \\
(\text { GM) }\end{array}$ \\
\hline CADC & Fonds FFTQ & MRC \\
CEIC & Fonds de capital de risque & Ville \\
Commission de la formation & Fonds Desjardins & \\
professionnelle & SOFICAR & \\
Club de recherche d'emploi & & \\
Revenu Canada & & \\
\hline
\end{tabular}

\begin{tabular}{|c|c|c|}
\hline $\begin{array}{l}\text { Entreprises } \\
\text { (EN) }\end{array}$ & $\begin{array}{l}\text { Gouvernement provincial } \\
\text { (GP) }\end{array}$ & $\begin{array}{l}\text { Regroupement d'affaires } \\
\text { (RA) }\end{array}$ \\
\hline $\begin{array}{l}\text { Assureur } \\
\text { Notaire } \\
\text { Avocat } \\
\text { Comptable } \\
\text { Spécialiste en marketing } \\
\text { Spécialiste en publicité } \\
\text { Conseiller généraliste } \\
\text { Économiste } \\
\text { Entrepreneur non professionnel }\end{array}$ & $\begin{array}{l}\text { Ministère de l'Industrie et } \\
\text { du Commerce } \\
\text { Bureau de député } \\
\text { Ministère des Communautés } \\
\text { culturelles } \\
\text { Commission du travail } \\
\text { du Québec } \\
\text { CIVT } \\
\text { Centre Travail Québec }\end{array}$ & $\begin{array}{l}\text { Chambre de commerce } \\
\text { Jeune Chambre de commerce } \\
\text { Association des femmes d'affaires } \\
\text { du Québec } \\
\text { Association des travailleurs } \\
\text { autonomes du Québec } \\
\text { Association des gens d'affaires } \\
\text { du centre-ville d'Amos } \\
\text { Association des directeurs de caisses }\end{array}$ \\
\hline
\end{tabular}

Spécialiste en ressources humaines SQDM

Spécialiste en communication SDI 
TABLEAU 2 (suite)

\section{Les commissions scolaires et leurs contacts socio-organisationnels}

\begin{tabular}{lll}
\hline $\begin{array}{l}\text { Entreprises } \\
\text { (EN) }\end{array}$ & $\begin{array}{l}\text { Gouvernement provincial } \\
(\text { GP) }\end{array}$ & $\begin{array}{l}\text { Regroupement d'affaires } \\
(\mathbf{R A})\end{array}$ \\
\hline $\begin{array}{l}\text { Spécialiste en gestion du temps } \\
\text { Spécialiste en informatique }\end{array}$ & $\begin{array}{l}\text { Table d'éducation et } \\
\text { de main-d'œure }\end{array}$ \\
$\begin{array}{l}\text { Spécialiste en vente } \\
\text { Spécialiste en ressources physiques }\end{array}$ & OPDQ & CREDQ \\
Spécialiste en financement & Secrétariat des affaires régionales \\
Spécialiste en négociation & CSST & \\
& Normes du travail du Québec & \\
\hline & Médias (ME) & Centres d'expertise (CE) \\
\hline & Journal écrit & Centre d'expertise touristique \\
& (hebdo, quotidien) & des Laurentides \\
& Radio & Association touristique \\
& Télévision grand réseau & Agence touristique \\
& Télévision communautaire & Centre de technologie \\
& Télévision locale & Info-accès (étude de marché) \\
& Télévision régionale & OCTAR \\
& & CARA \\
\hline
\end{tabular}

Nous retrouvons les institutions financières (IF), les corporations de développement économique (CD), les groupes de soutien technique à l'entreprise (GS), les organisations scolaires (OS), les entreprises (EN), les organismes communautaires (OC), les centres d'expertise (CE), les fonds de financement (FF), les regroupements du milieu des affaires (RA), les médias (ME) et, finalement, les gouvernements fédéral (GF), provincial (GP) et municipal (GM). Ce premier découpage introduit un deuxième niveau d'analyse contenu dans les données du tableau 3.

En effet, l'examen attentif du nombre de contacts que les commissions scolaires ont établis avec les organisations de leur milieu nous amène à relever deux points.

Premièrement, les commissions scolaires ont pris l'initiative de 1038 contacts avec les 96 organisations durant le programme. Les entreprises sont bonnes premières avec 293 contacts $(28,2 \%)$ suivies de loin par les institutions financières avec 133 contacts $(12,8 \%)$ et les groupes de soutien avec 123 contacts $(11,8 \%)$. Dans un autre sous-groupe, nous identifions les corporations de développement avec 81 contacts $(7,8 \%)$, les médias, 78 contacts $(7,5 \%)$, le gouvernement fédéral, 64 contacts $(6,2 \%)$, les organisations communautaires, 62 contacts $(6 \%)$ et le gouvernement provincial avec 58 contacts $(5,6 \%)$. Dans le peloton de queue, nous retrouvons les regroupements de gens d'affaires avec 50 contacts $(4,8 \%)$, le gouvernement municipal, 36 contacts $(3,5 \%)$, les organisations scolaires, 34 contacts (3,3\%) et, enfin, les centres d'expertise avec 13 contacts $(1,25 \%)$ et les fonds de financement aussi avec 13 contacts $(1,25 \%)$. 
TABLEAU 3

Nombre de contacts des commissions scolaires avec les organisations du milieu

\begin{tabular}{|c|c|c|c|c|c|c|c|c|c|c|c|c|c|c|}
\hline & $\begin{array}{l}\text { Institutions } \\
\text { financières }\end{array}$ & $\begin{array}{l}\text { Corpora- } \\
\text { tions de } \\
\text { dévelop- } \\
\text { pement }\end{array}$ & $\begin{array}{c}\text { Groupes } \\
\text { de } \\
\text { soutien }\end{array}$ & $\begin{array}{l}\text { Organi- } \\
\text { sations } \\
\text { scolaires }\end{array}$ & Entreprises & $\begin{array}{c}\text { Organi- } \\
\text { sations } \\
\text { commu- } \\
\text { nautaires }\end{array}$ & $\begin{array}{c}\text { Centres } \\
\text { d'expertise }\end{array}$ & $\begin{array}{l}\text { Fonds } \\
\text { de } \\
\text { finan- } \\
\text { cement }\end{array}$ & $\begin{array}{c}\text { Regrou- } \\
\text { pement } \\
\text { d'affaires }\end{array}$ & Médias & $\begin{array}{l}\text { Gouver- } \\
\text { nement } \\
\text { fédéral }\end{array}$ & $\begin{array}{c}\text { Gouver- } \\
\text { nement } \\
\text { provincial }\end{array}$ & $\begin{array}{c}\text { Gouver- } \\
\text { nement } \\
\text { municipal }\end{array}$ & Total \\
\hline $\begin{array}{l}\text { Obtention } \\
\text { de l'ASP }\end{array}$ & 21 & 10 & 16 & 2 & 9 & 2 & 1 & 3 & 10 & 0 & 11 & 5 & 19 & 109 \\
\hline $\begin{array}{l}\text { Planification } \\
\text { budgétaire }\end{array}$ & 0 & 2 & 3 & 0 & 8 & 1 & 1 & 1 & 1 & 0 & 2 & 2 & 3 & 24 \\
\hline $\begin{array}{l}\text { Organisation } \\
\text { des locaux }\end{array}$ & 0 & 0 & 1 & 0 & 6 & 1 & 0 & 0 & 0 & 0 & 0 & 0 & 0 & 8 \\
\hline $\begin{array}{l}\text { Choix du } \\
\text { coordonnateur }\end{array}$ & 7 & 1 & 3 & 1 & 1 & 1 & 0 & 0 & 2 & 1 & 0 & 1 & 2 & 20 \\
\hline $\begin{array}{l}\text { Promotion } \\
\text { du programme }\end{array}$ & 41 & 28 & 43 & 20 & 55 & 52 & 5 & 8 & 22 & 38 & 37 & 29 & 12 & 390 \\
\hline $\begin{array}{l}\text { Recrutement } \\
\text { des candidats }\end{array}$ & 24 & 17 & 14 & 9 & 13 & 3 & 3 & 1 & 7 & 39 & 8 & 4 & 0 & 142 \\
\hline $\begin{array}{l}\text { Planification } \\
\text { pédagogique }\end{array}$ & 9 & 4 & 8 & 0 & 51 & 1 & 0 & 0 & 1 & 0 & 2 & 2 & 0 & 78 \\
\hline $\begin{array}{l}\text { Enseignement } \\
\text { en classe }\end{array}$ & 24 & 10 & 15 & 1 & 105 & 0 & 2 & 0 & 6 & 0 & 3 & 13 & 0 & 179 \\
\hline $\begin{array}{l}\text { Évaluation } \\
\text { des apprentissages }\end{array}$ & 3 & 2 & 9 & 0 & 39 & 0 & 0 & 0 & 0 & 0 & 0 & 1 & 0 & 54 \\
\hline Total & 133 & 81 & 123 & 34 & 293 & 62 & 13 & 13 & 50 & 78 & 64 & 58 & 36 & 1038 \\
\hline
\end{tabular}


Deuxièmement, l'ouverture des commissions scolaires vers le milieu a été plus marquée lors des étapes de la promotion du programme (390 contacts), de l'enseignement en classe (179 contacts), de recrutement des candidats (142 contacts) et de l'obtention de l'ASP (109 contacts). Les étapes de la planification budgétaire (24 contacts), de l'organisation des locaux (huit contacts), du choix du coordonnateur-enseignant ( 20 contacts) et de la sélection des candidats (34 contacts) demeurent grosso modo l'affaire des commissions scolaires.

Cette analyse ne doit pas nous faire oublier que les entreprises sont de toutes façons les organisations les plus nombreuses dans un milieu, contrairement à un gouvernement municipal, à un centre d'expertise ou à un fonds de financement. Nous verrons plus loin que la fréquence des contacts socio-organisationnels d'une commission scolaire ne suffit pas pour quantifier leurs interactions, encore faut-il estimer la qualité ou l'intensité de ces interactions.

\section{Valeur des interactions socio-organisationnelles (VISO)}

En croisant les 13 catégories d'organisations avec les 10 étapes du processus d'implantation décrites plus haut, force est de constater que la participation des organisations qui gravitent autour des commissions scolaires varie selon les étapes du processus. Mais comment évaluer le degré d'intensité d'une interaction qui se vit à l'intérieur des limites du programme ? La réponse nous vient d'une analyse détaillée des documents d'accompagnement du MEQ. Selon ces sources, les 10 étapes du processus d'implantation n'ont pas la même intensité. Elles se caractérisent par un des trois niveaux (un à trois) selon l'intensité souhaitée par le programme cadre. Rappelons que le niveau 1 désigne une interaction d'information entre l'école et le milieu, le niveau 2, l'appui de l'organisation communautaire à la formation et sa solidarité à l'égard du programme, et le niveau 3, la collaboration étroite entre l'école et l'organisation du milieu pour implanter la formation. Voici donc, dans les prochaines lignes, les valeurs correspondantes des interactions entre l'école et le milieu selon les 10 étapes du processus d'implantation du programme.

\begin{tabular}{|c|c|c|c|c|}
\hline Étape 1 & $-<$ & Obtention de l'ASP & $\longrightarrow$ & Niveau 2 \\
\hline Étape 2 & 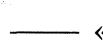 & Planification budgétaire & $\longrightarrow$ & Niveau 1 \\
\hline Étape 3 & - & Organisation des locaux & & Niveau 3 \\
\hline Étape 4 & 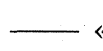 & Choix du coordonnateur & & Niveau 3 \\
\hline Étape 5 & $<$ & Promotion du programme & & Niveau 1 \\
\hline Étape 6 & $<$ & Recrutement des candidats & & Niveau 2 \\
\hline Étape 7 & $<$ & Sélection des candidats & & Niveau 3 \\
\hline Étape 8 & $<$ & Planification pédagogique & & Niveau 3 \\
\hline Éta & $<$ & Enseignement en classe & & Nive \\
\hline Étape 10 & 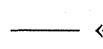 & Évaluation des apprentissages & $\longrightarrow$ & Niveau 3 \\
\hline
\end{tabular}


En fait, la démarche est simple: tout d'abord, nous relevons le nombre de contacts que l'école a entretenu avec les 13 catégories d'organisations, et ce, tout au long des 10 étapes du processus. Puis chaque sous-total de contacts par étape est pondéré par sa valeur correspondante. Le VISO est la somme de toutes ces opérations. Nous présentons les 16 VISO au graphique 1.

\section{GRAPHIQUe 1}

\section{Valeur des interactions socio-organisationnelles (VISO)}

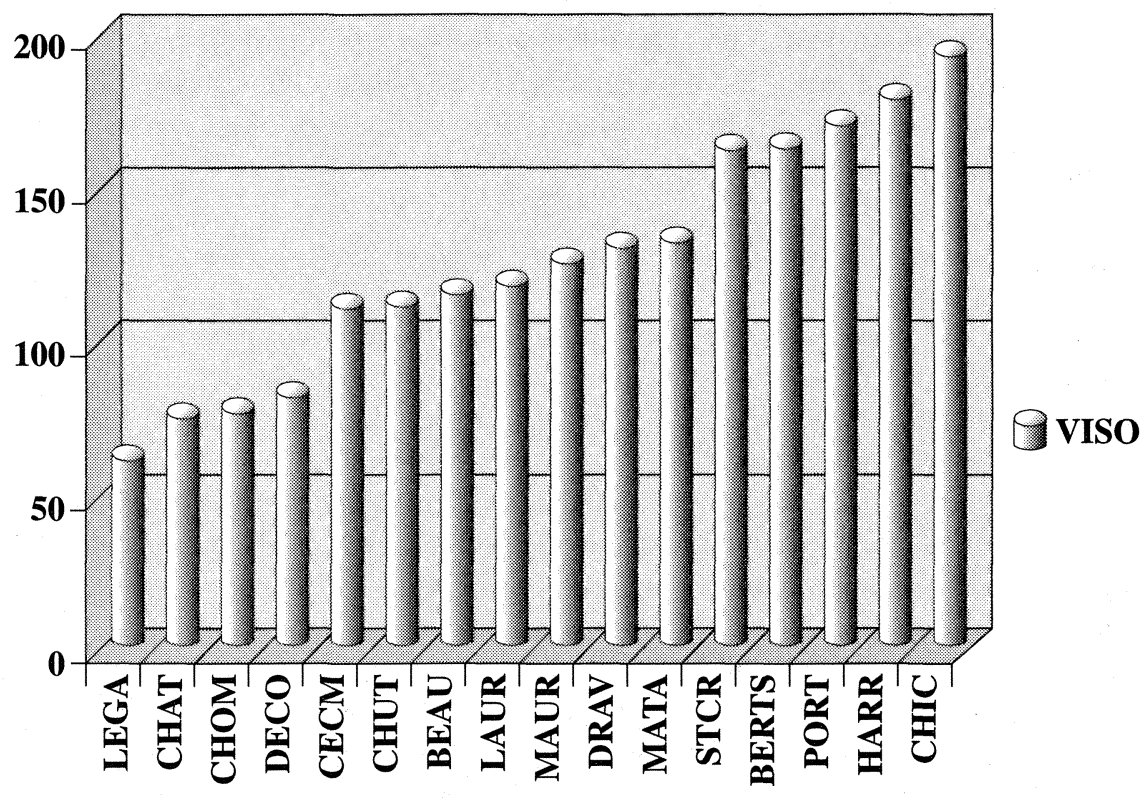

Ces résultats indiquent une distribution du VISO s'échelonnant de 61 à 190. Nous retrouvons les commissions scolaires Le Gardeur (LEGA), Châteauguay (CHAT), Chomedey (CHOM), et Découvreurs (DECO) qui ont entretenu de faibles interactions avec leur milieu (VISO $\leq 100$ ). Puis nous observons des interactions moyennes (VISO entre 101 et 150) chez les commissions scolaires telles que la CECM, Chutes-de-la-Chaudière (CHUT), Beauport (BEAU), Laurentides (LAUR), Mauricie (MAUR), Draveurs (DRAV) et Vallée-de-la-Matapédia (MATA). Finalement, nous identifions dans une troisième classe les commissions scolaires Sainte-Croix (STCR), Bersimis (BERS), Portneuf (PORT), Harricana (HARR) et Chicoutimi (CHIC) qui ont entretenu des interactions élevées avec leur milieu (VISO > 150). De quelle façon pouvons-nous analyser ces trois types de comportements organisationnels? 


\section{Trois groupes distincts}

L'agrégation des scores VISO (en \%) selon les trois catégories mentionnées ci-dessus et leur analyse par rapport aux 10 étapes du processus d'implantation du programme font ressortir des résultats intéressants (voir le tableau 4).

TABLEAU 4

Répartition des interactions (\%) selon les trois catégories de VISO

\begin{tabular}{lrcr}
\hline & VISO faible & VISO moyen & VISO élevé \\
\hline 1. Obtention de l'ASP & $12,5 \%$ & $9,0 \%$ & $11,7 \%$ \\
2. Planification budgétaire & $1,6 \%$ &, $35 \%$ & $1,8 \%$ \\
3. Organisation des locaux & $2,9 \%$ & $1,4 \%$ &, $3 \%$ \\
4. Choix du coordonnateur & $2,0 \%$ & $4,9 \%$ & $1,4 \%$ \\
5. Promotion du programme & $13,1 \%$ & $20,3 \%$ & $20,0 \%$ \\
6. Recrutement des candidats & $5,9 \%$ & $12,8 \%$ & $17,9 \%$ \\
7. Sélection des candidats & $3,9 \%$ & $7,7 \%$ & $2,8 \%$ \\
8. Planification pédagogique & $11,8 \%$ & $7,0 \%$ & $15,8 \%$ \\
9. Enseignement en classe & $43,3 \%$ & $25,8 \%$ & $21,4 \%$ \\
10. Évaluation des apprentissages & $3,0 \%$ & $10,8 \%$ & $6,9 \%$ \\
\hline Total (\%) & $\mathbf{1 0 0 , 0} \%$ & $\mathbf{1 0 0 , 0} \%$ & $\mathbf{1 0 0 , 0} \%$ \\
\hline
\end{tabular}

Les écoles avec un VISO faible mettent le gros de leurs énergies à ouvrir la période d'enseignement à d'autres formateurs du milieu (43,3\%), laissant peu de place aux interactions durant les autres étapes du processus d'implantation. Les entreprises (EN) sont celles qui bénéficient le plus de cette ouverture. Ainsi, il n'est pas rare de voir dans la classe des assureurs, des notaires, des comptables, des avocats, des spécialistes en marketing, en publicité, en ressources humaines, en communication, en gestion du temps, en informatique, en ventes, en ressources physiques, en financement, en négociation, quelques économistes et surtout beaucoup d'entrepreneurs. Les institutions financières (IF) sont aussi bien représentées dans les classes.

Quand les écoles présentent un VISO moyen, elles valorisent toujours l'ouverture de la classe, mais elles sont aussi préoccupées par la promotion du programme. Elles veulent s'assurer que l'ensemble des partenaires du milieu contribuent à la diffusion de l'information concernant le programme de formation en entrepreneuriat. Les entreprises (EN), les organisations communautaires (OC), les groupes de soutien à l'entrepreneuriat (GS) et les institutions financières (IF) sont les plus actives dans ce dossier. 
Finalement, les écoles avec un MIS élevé présentent non seulement une ouverture de la classe et une diffusion de l'information, mais elles valorisent des interactions pour le recrutement des candidats et la planification pédagogique. Sur le plan du recrutement, les médias (ME) écrits et électroniques se chargent d'attirer des entrepreneurs potentiels aux soirées d'information, tandis que sur le plan de la planification pédagogique, les entreprises $(\mathrm{EN})$ mettent la main à la pâte en influençant les décisions reliées à la structuration des objectifs, aux éléments de contenu, aux stratégies d'enseignement-apprentissage et aux critères d'évaluation.

Mais comment peut-on interpréter tous ces résultats et les relier aux connaissances antérieures dans le domaine de l'entrepreneuriat et de l'éducation ? La dernière section fait le point sur cette question.

\section{Discussion}

Tout d'abord, cette recherche a permis d'identifier les 10 étapes du processus d'implantation d'un programme de formation offert dans 16 commissions scolaires du Québec. À notre avis, il n'y a pas d'autres recherches en entrepreneuriat et éducation qui ont permis de décortiquer un tel processus. Puis, nous avons mis en relief 13 catégories distinctes d'organisations qui gravitent autour du programme de formation en entrepreneuriat. L'intérêt de ces résultats est de classer les différentes organisations en catégories facilement identifiables. Seules les organisations religieuses rapportées par Welsch (1993) n'apparaissent pas dans nos résultats.

Par la construction d'une mesure de la valeur des interactions socio-organisationnelles (VISO), nous avons pu estimer les interactions que l'école entretient avec le milieu. Cela ne semble pas avoir été soulevé dans la littérature sur l'entrepreneuriat et l'éducation. Ainsi, cette démarche a permis de mettre au jour trois types de comportements organisationnels : des commissions scolaires avec un VISO faible, moyen et élevé selon le degré d'ouverture et de partenariat qu'elles entretiennent avec leur milieu. Une analyse attentive des résultats révèle que plus une institution scolaire s'ouvre sur le milieu, plus elle le fait à plusieurs moments du processus d'implantation du programme. Une école, un collège ou une université ouverte sur le milieu permet aux ressources éducatives qui l'entourent d'intervenir non seulement pour l'enseignement en classe, mais lors de la promotion du programme, lors du recrutement des candidats et aussi lors de l'étape de la planification pédagogique.

Mais quelle est donc l'utilité de ces résultats ? Tout d'abord, selon une perspective des ressources humaines, cette recherche met en évidence le rôle fondamental de l'enseignant-coordonnateur. Cet acteur a la responsabilité non seulement d'attirer les ressources du milieu, mais de favoriser leur implication dans le programme de formation (Robinsen et Christensen 1992). On peut raisonnablement penser que plus 
cette personne est reliée à son milieu, plus le programme en bénéficie. L'histoire de l'enseignant-coordonnateur de la commission scolaire de Châteauguay démontre bien l'importance du choix de ce dernier. Lors de l'entrevue, nous avons appris qu'il avait exercé les fonctions de commissaire industriel dans une région du Nord du Québec avant d'accepter ce poste dans le Sud du Québec. Les résultats du VISO de Châteauguay laissent entendre que, malgré une disposition à travailler avec le milieu, cet enseignant-coordonnateur connaissait peu ou pas les gens de cette nouvelle région. Le programme a conséquemment peu retiré des ressources régionales. Choisir un acteur connu du milieu et reconnu pour ses compétences devient un impératif.

Sur le plan de l'école, les résultats illustrent bien la démarche des 16 commissions scolaires retenues dans le programme provincial. Les directeurs généraux de ces institutions répondent en fait à l'avis lancé en 1989 par le Conseil supérieur de l'éducation au ministre de l'époque voulant que le développement socioéconomique régional soit aussi l'affaire des gens de l'Éducation (Conseil supérieur de l'éducation, 1989). L'appel aux décloisonnements des mandats de tous et de chacun fait en sorte que l'école veut et peut intervenir au chapitre de l'économie et vice versa pour les autres partenaires dans les affaires éducatives. Au lieu de former des jeunes pour une insertion professionnelle ultérieure, le cas de l'entrepreneuriat interpelle les institutions d'enseignement à valoriser l'esprit d'entreprise en collaboration avec tous les acteurs de la scène régionale. L'école partage donc son pouvoir d'expertise avec d'autres acteurs de la société. Là encore, les résultats du VISO dénotent une diversité de comportements à ce chapitre.

Concernant les ressources communautaires, on peut s'interroger sur la motivation de chacune d'entre elles à s'engager dans le programme de formation en entrepreneuriat. En d'autres termes, quel est le but réel visé par ces leaders? S'impliquent-ils pour bien paraître, parce qu'ils n'ont pas le choix ou parce qu'ils croient en l'initiative proposée ? En fait, cette recherche a tenté de mesurer non pas la motivation des acteurs (vouloir s'engager, pouvoir s'engager) ni le contexte régional d'engagement, mais le comportement tel quel. Ce qui signifie qu'il se peut que certains leaders socioéconomiques se soient impliqués pour des motifs personnels, opportunistes ou autres. En ce sens, VISO ne rend pas compte de l'aspect persévérance (le long terme) du comportement. En effet, l'engagement et la persévérance sont deux notions importantes pour s'assurer que l'information circulant dans le milieu se transforme en information structurante comme l'énonce Laborit dans Julien (1997). La mesure VISO doit donc être comprise dans un espace-temps court terme. Par contre, il serait intéressant de reprendre une deuxième mesure de la valeur des interactions socio-organisationnelles pour vérifier justement la persévérance des organisations de chaque milieu dans l'effort de formation des entrepreneurs. On aurait là une plus juste idée des effets structurants des interactions école-milieu. 
En terminant, rappelons que cette recherche a toutes les qualités et les défauts d'une approche qualitative. Ainsi, la précision des données du seul cas du Québec amène à la prudence quant à la validité externe de nos résultats. En effet, la mesure VISO doit être éprouvée dans d'autres circonstances pour en assurer la robustesse. Finalement, on ne sait toujours pas s'il y a un lien entre l'ouverture de l'école et le taux de création d'entreprises. D'autres variables telles que la nature du contenu à enseigner, le degré de motivation des entrepreneurs potentiels, ainsi que la prestation des formateurs sont à considérer pour mieux comprendre l'univers des programmes de formation en entrepreneuriat.

\section{Bibliographie}

ALDRICH, H. (1976b), « Resource dependance and inter-organizational relations », Administration and Society, vol. 7, p. 419-454.

BROCKHAUS, R. H. Sr. (1991), «Entrepreneurship education and research outside North America », Entrepreneurship, Theory and Practice, vol. 15, no 3, p. 77-84.

CARSRUD, A. L. (1991), «Entrepreneurship and enterprise formation : a brief perspective on the infrastructure in Europe », Entrepreneurship Theory and Practice, printemps, vol. $15, \mathrm{n}^{\circ} 3$, p. $69-75$.

Conseil supérieur de l'éducation (1989), Le développement socio-économique régional: un choix à raffermir en éducation, Avis aux ministre de l'Éducation et ministre de l'Enseignement supérieur et de la Science, Gouvernement du Québec.

CHRISTY, R. et B. M. JONES (1982), The Complete Information Bank for Entrepreneurs and Small Business Managers, Wichitor, K. S. : Center for Entrepreneurship and Small Business Management at Wichita State University.

DANA, L. P. (1990), « Towards an integrated needs - related policy on entrepreneurship », Canadian Journal of Administrative Sciences, vol. 7, $\mathrm{n}^{\circ}$ 2, p. 25-33.

DART, J. et L. L. PENDLETON (1984), « The role of advertising agencies in entrepreneurial education », Journal of Small Business Management, avril, vol. 22, $\mathrm{n}^{\circ} 2$, p. 38-44.

Delaney, J. (1988), «Making a difference», Venture, numéro de décembre, p. 67-70.

Direction générale de la formation professionnelle (1991b), "Lancement d'une entreprise », Rapport de la réunion du comité consultatif (document de travail), Ministère de l'Éducation du Québec, Administration, Commerce et Secrétariat.

Direction générale de la formation professionnelle, ministère de l'Éducation du Québec (1991c), Orientations de l'attestation de spécialisation professionnelle (ASP), «Lancement d'une entreprise».

Gatewood, E., S. MiRanda et F. Hoy (1990), « The involvement of private foundations in entrepreneurial research », Journal of Small Business Management, vol. 28, $\mathrm{n}^{\circ} 2$, p. 20-29.

GIBB, A. et G. MANU (1990), «Design of extension and support services for small-scale enterprise development », International Small Business Journal, vol. 8, no 3, p. 10-26. 
HALL, R. C. (1987), Organizations Structure and Process, Englewoods Cliffs, New Jersey, Prentice-Hall Inc.

JULIEN, P. A. (sous la direction de), (1997), Les PME: bilan et perspectives, $2^{2}$ édition, Cap-Rouge, Presses Inter Universitaires ; Paris, Economica.

KATZ, J. A. (1991), «The institution and infrastructure of entrepreneurship », Entrepreneurship Theory and Practice, vol. 15, $\mathrm{n}^{\circ} 3$, p. 85-104.

KATZ, J. A. (1993), Growth of Endowments Chairs and Programs in Entrepreneurship on the College Campus, Project for Excellence in Entrepreneurship Education, BaldwinWallace College, Cleveland, Ohio.

KIERULFF, H. E. (1985), Entrepreneurship Chairs and Centers, Seattle, Seattle Pacific University / International Council for Small Business.

LECLERC, W. (1985), «Universities and entrepreneurs », Journal of Small Business and Entrepreneurship, vol. 3, no 2, p. 41-47.

MARRETT, C. B. (1971), « On the specification of inter-organizational dimensions », Sociology and Social Research, vol. 56, p. 83-99.

Moles, A. (1975), « Systèmes de média et systèmes éducatifs », Perspectives, UNESCO, vol. $6, \mathrm{n}^{0} 2$, p. 176-188.

RoBINSON, P. B. et M. A. CHRISTENSEN (1992), L'enseignement de l'entrepreneurship au Canada, Rapport établi pour le compte du deuxième Colloque national INDE sur l'enseignement de l'entrepreneurship, Moncton, Nouveau-Brunswick, sous la direction de l'Institut national de développement de l'entrepreneurship et le Bureau de l'entrepreneurship et de la petite entreprise, Industrie, Sciences et Technologie Canada.

ROBINSON, P. et M. HAYNES (1991), «Entrepreneurship education in America's major universities », Entrepreneurship Theory and Practice, vol. 15, no 3, p. 41-52.

SANDBERG, W. R. et E. J. GATEWOOD (1991), «A profile of entrepreneurship research centers : orientations interests, activities, and resources », Entrepreneurship Theory and Practice, vol. 15, $\mathrm{n}^{\circ} 3$, p. 11-23.

SOLOMON, G. et M. CARNEY (1985), «United States small business administration's role in small business training », International Small Business Journal, vol. 4, n 1, p. 25-32.

WELSCH, H. D. (1993), The Infrastructure of Entrepreneurship Education, Project for Excellence in Entrepreneurship Education, Baldwin-Wallace College, Cleveland, Ohio. 